\title{
Temperature-dependent dielectric and piezoelectric response of ferroelectrics from first principles ${ }^{1}$
}

\author{
K. M. Rabe and E. Cockayne \\ Department of Applied Physics, Yale University \\ P. O. Box 208284, New Haven, Connecticut, 06520-8284
}

\begin{abstract}
A method for the calculation of the temperature dependence of dielectric and piezoelectric responses, based on the use of a first-principles effective Hamiltonian, is described. Results are presented for the ferroelectric perovskite $\mathrm{PbTiO}_{3}$. While the method includes only the soft-mode contributions to the responses, it is argued to give a good description of the divergences or near-divergences of the response functions near the cubic-tetragonal transition. The expression of the response functions in terms of correlation functions is used to provide a real-space interpretation of the responses which clearly distinguishes between $\mathrm{PbTiO}_{3}$ and the related materials $\mathrm{BaTiO}_{3}$ and $\mathrm{KNbO}_{3}$.
\end{abstract}

\section{INTRODUCTION}

Systems with large dielectric and piezoelectric responses are of interest both from a fundamental and a technological point of view [1]. Understanding the microsopic origin of a high sensitivity of the polarization of a ferroelectric oxide to applied field, in the case of the dielectric constant, or stress-induced changes in strain, in the case of piezoelectricity, can lead to the optimization of these properties through the appropriate choice of materials. Empirically, large responses are observed in stoichiometric compounds such as $\mathrm{PbTiO}_{3}$ and $\mathrm{BaTiO}_{3}$ for temperatures near the ferroelectric phase transition [2-5]. In solid solutions, such as PZT, enhanced responses are observed over a wide temperature range [6].

In this paper, we discuss the modeling of the piezoelectric and dielectric response of ferroelectric perovskites, specifically $\mathrm{PbTiO}_{3}$ and $\mathrm{BaTiO}_{3}$, though the use of first-principles effective Hamiltonians previously constructed in Refs. [7-10]. The calculations give reasonable agreement with experiment, considering that this modeling gives only the contribution of the soft modes to the responses, and not

1) To be published in the proceedings of the Fifth Williamsburg Workshop on First-Principles Calculations for Ferroelectrics, February 1998. 
other contributions such as thermal expansion and the response of other polar modes. However, for temperatures near the ferroelectric phase transition, the soft modes are expected to dominate and thus this approach should capture the essential physics of the large observed responses. In addition, it should correctly describe the trends between different materials. Finally, with this microscopic approach, we are able directly to relate the calculated response functions to the characteristic correlations of local unit cell polarizations, which should yield further insight into the nature of the large responses of interest.

\section{FIRST-PRINCIPLES EFFECTIVE HAMILTONIANS}

The construction of first-principles effective Hamiltonians for $\mathrm{PbTiO}_{3}$ and $\mathrm{BaTiO}_{3}$ has previously been described in detail [8-10]. Briefly, the model consists of one vector per five-atom unit cell which represents the local polarization associated with the ferroelectric distortion. The potential $\mathcal{H}_{e f f}\left(\left\{\vec{\xi}_{i}\right\}, e_{\alpha \beta}\right)$ for these vector degrees of freedom is expanded for the high-symmetry cubic perovskite reference structure, including local anharmonic terms, quadratic intersite interactions which are assumed to be dipolar beyond third neighbors, and lowest order coupling to homogeneous strain.

The extension of this model to include the effects of electric field is accomplished by writing the dependence of the polarization on the model degrees of freedom to lowest order:

$$
P\left(\left\{\vec{\xi}_{i}\right\}, e_{\alpha \beta}\right)=\sum_{i} \bar{Z}^{*} e a_{0} \vec{\xi}_{i}
$$

and adding to the potential the coupling term $-\vec{P} \cdot \vec{E}$ where $\vec{E}$ is the macroscopic electric field [11]. The dielectric response is then simply obtained from $\frac{d<P_{\alpha}>}{d E_{\beta}}$, while the piezoelectric response can be expressed as $\frac{\left.d<e_{\alpha \beta}\right\rangle}{d E_{\gamma}}$, where the brackets are used to denote the thermal expectation value. The effects of macroscopic stress $\sigma_{i j}$ can be included by adding to the potential the coupling term $-\sigma_{i j} \cdot e_{i j}$. With these couplings, the thermodynamic identity $\frac{d e_{j k}}{d E_{i}}=\frac{d P_{i}}{d \sigma_{j k}}$ can be obtained (the wellknown equality of the direct and converse piezoelectric effects [11]). In the rest of this paper, we work at zero external stress and evaluate the piezoelectric response using $\frac{d<e_{\alpha \beta}>}{d E_{\gamma}}$.

Calculations of the temperature dependent properties of the system are carried out using classical Monte Carlo calculations, as previously described [8,9]. The calculations of the response functions presented here involved $7 \times 7 \times 7$ simulation cells, 10,000 Monte Carlo sweeps (MCS) for thermalization, 200,000 MCS for the computation of thermal expectation values, and several runs at each temperature with different random number seeds to estimate statistical error. The calculations of the real-space correlation functions presented here involved 10x10x10 simulation cells, 10,000 Monte Carlo sweeps (MCS) for thermalization, and 100,000 MCS for the 
computation of thermal expectation values. In order to compute the temperaturedependence of the order parameter and thus identify the cubic-tetragonal transition, a small symmetry-breaking field $E_{z}$ of magnitude $\frac{20}{\epsilon_{\infty}} \mathrm{kV} / \mathrm{cm}$ was applied. The response function results include this nonzero field.

\section{CORRELATION FUNCTION EXPRESSIONS FOR RESPONSE FUNCTIONS}

In the Monte Carlo simulations described above, the response functions are computed using the fact that they can be expressed as correlation functions. This allows the appropriate derivatives to be calculated in one Monte Carlo run.

Specifically, we consider the dielectric tensor:

$$
\epsilon_{\alpha \beta}=\epsilon_{\infty}+4 \pi \chi_{\alpha \beta}
$$

where

$$
\chi_{\alpha \beta}=\frac{\partial<\Omega^{-1} P_{\alpha}>}{\partial E_{\beta}} .
$$

Here, $\Omega$ is the volume of the unit cell and $\vec{P}$ is the polarization per unit cell, specifically

$$
<P_{i \alpha}>=\frac{\int\left\{d e_{\alpha \beta}\right\}\left\{\prod_{j} d \vec{\xi}_{j}\right\}\left(\frac{Z^{*} e a_{0}}{N} \sum_{i} \xi_{i \alpha}\right) \exp \left(-\beta\left(H_{e f f}-Z^{*} e a_{0} \sum_{i} \xi_{i \alpha} \cdot \vec{E}\right)\right)}{\int\left\{d e_{\alpha \beta}\right\}\left\{\prod_{j} d \vec{\xi}_{j}\right\} \exp \left(-\beta\left(H_{e f f}-Z^{*} e a_{0} \sum_{i} \xi_{i \alpha} \cdot \vec{E}\right)\right)}
$$

or

$$
<P_{i \alpha}>=\left(\frac{1}{N \beta}\right) \frac{\partial \ln Z(\beta, \vec{E})}{\partial E_{\alpha}}
$$

where we have defined

$$
Z(\beta, \vec{E})=\int\left\{d e_{\alpha \beta}\right\}\left\{\prod_{j} d \vec{\xi}_{j}\right\} \exp \left(-\beta\left(H_{e f f}-Z^{*} e a_{0} \sum_{i} \xi_{i \alpha} \cdot \vec{E}\right)\right) .
$$

Using the approximation $\frac{\partial\left\langle\Omega^{-1} P_{\alpha}\right\rangle}{\partial E_{\beta}}=\Omega^{-1} \frac{\partial\left\langle P_{\alpha}\right\rangle}{\partial E_{\beta}}$ (see footnote 7 in Ref. [12]) and differentiating Equation 2 with respect to $E_{\alpha}$, we readily find the correlation function expression:

$$
\chi_{\alpha \beta}=\frac{\beta\left(Z^{*} e a_{0}\right)^{2}}{\Omega}\left(<\sum_{i} \xi_{i \alpha} \frac{1}{N} \sum_{j} \xi_{j \beta}>-N<\xi_{\alpha}><\xi_{\beta}>\right)
$$

where

$$
<\xi_{\alpha}>=\frac{1}{N}<\sum_{i} \xi_{i \alpha}>
$$


The piezoelectric tensor can be expressed in terms of correlation functions in an completely analogous way:

$$
d_{\gamma \alpha \beta}=\frac{\partial<e_{\alpha \beta}>}{\partial E_{\gamma}}
$$

where

$$
<e_{\alpha \beta}>=\frac{\int\left\{d e_{\alpha^{\prime} \beta^{\prime}}\right\}\left\{\prod_{j} d \vec{\xi}_{j}\right\} e_{\alpha \beta} \exp \left(-\beta\left(H_{e f f}-Z^{*} e a_{0} \sum_{i} \vec{\xi}_{i} \cdot \vec{E}\right)\right)}{\int\left\{d e_{\alpha^{\prime} \beta^{\prime}}\right\}\left\{\prod_{j} d \vec{\xi}_{j}\right\} \exp \left(-\beta\left(H_{e f f}-Z^{*} e a_{0} \sum_{i} \vec{\xi}_{i} \cdot \vec{E}\right)\right)} .
$$

Differentiating Equation 4 with respect to $E_{\gamma}$, we find the correlation function expression:

$$
d_{\gamma \alpha \beta}=\beta\left(Z^{*} e a_{0}\right)\left(<e_{\alpha \beta} \sum_{j} \xi_{j \gamma}>-N<e_{\alpha \beta}><\xi_{\gamma}>\right) .
$$

When the homogeneous strain appears only up to quadratic order in the effective Hamiltonian, as is the case for the effective Hamiltonians available for $\mathrm{PbTiO}_{3}[8,9]$, $\mathrm{BaTiO}_{3}[10]$ and $\mathrm{KNbO}_{3}$ [13], the piezoelectric tensor can be reexpressed in terms of correlations of local polar distortions. Because the integral over strain is Gaussian, completely equivalent expressions for the thermal expectation values involving $e_{\alpha \beta}$ can be obtained by making the following substitutions (and cyclic permutations thereof):

$$
\begin{gathered}
e_{x x} \rightarrow-\frac{1}{N}\left(\frac{\left(g_{0}+\frac{g_{1}}{3}\right)}{\left(C_{11}+2 C_{12}\right)} \sum_{i}\left|\vec{\xi}_{i}\right|^{2}+\frac{g_{1}}{3\left(C_{11}-C_{12}\right)} \sum_{i}\left(2 \xi_{i x}^{2}-\xi_{i y}^{2}-\xi_{i z}^{2}\right)\right) \\
e_{x y} \rightarrow-\frac{1}{N} \frac{g_{2}}{C_{44}} \sum_{i} \xi_{i x} \xi_{i y}
\end{gathered}
$$

For the example of $d_{33}$, this yields

$$
\begin{array}{r}
d_{33}=-\frac{\beta\left(Z^{*} e a_{0}\right)}{N}\left(\frac{\left(g_{0}+\frac{g_{1}}{3}\right)}{\left(C_{11}+2 C_{12}\right)} \sum_{i, j}\left(<\left|\vec{\xi}_{i}\right|^{2} \xi_{j z}>-<\left|\vec{\xi}_{i}\right|^{2}><\xi_{j z}>\right)\right. \\
+\frac{g_{1}}{3\left(C_{11}-C_{12}\right)} \sum_{i, j}\left(2\left(<\xi_{i x}^{2} \xi_{j z}>-<\xi_{i x}^{2}><\xi_{j z}>\right)\right. \\
-\left(<\xi_{i y}^{2} \xi_{j z}>-<\xi_{i y}^{2}><\xi_{j z}>\right) \\
\left.-\left(<\xi_{i z}^{2} \xi_{j z}>-<\xi_{i z}^{2}><\xi_{j z}>\right)\right)
\end{array}
$$

While Equation 5 proves more convenient for the actual computation of $d_{\gamma \alpha \beta}$, it is Equation 8 that naturally leads to a microscopic interpretation of the soft-mode response, as we will discuss further below. 


\section{$\epsilon_{z z}$ AND $d_{33}$ FOR $\mathbf{P b T i O}_{3}$}

The results of the Monte Carlo calculations for $\epsilon_{z z}$ are shown in Figure 1. At room temperature, the calculated value is 66 , which should be compared to the single-crystal measurement $\epsilon_{z z}=80$ [14]. The agreement is surprisingly good, considering that the calculation includes only the soft-mode contribution. The room temperature value is already considerably larger than the zero-temperature calculated value of 42 . The overall temperature dependence is very similar to that observed experimentally $[2,14]$, with a slow increase with temperature below the transition, a near-divergence both above and below $\mathrm{T}_{c}$ which is cut off by the firstorder character of the cubic-tetragonal transition, and an enhanced value of the dielectric response in the high-temperature cubic phase.

The results of the Monte Carlo calculations for $d_{33}$ are shown in Figure 2. At room temperature, the calculated value is 76 , which should be compared to the singlecrystal measurement $d_{33}=83.7$ [14]. As for the dielectric response, the agreement is surprisingly good, considering that the calculation includes only the soft-mode contribution. The room temperature value is already considerably larger than the zero-temperature calculated value of 48 . The overall temperature dependence is very similar to that observed experimentally $[2,4]$, with a slow increase with temperature below the transition, a near-divergence below $\mathrm{T}_{c}$ which is cut off by the first-order character of the cubic-tetragonal transition, and a drop to zero in the high-temperature cubic phase, as required by symmetry.

These results can be compared with the closely analogous calculation of the piezoelectric coefficients of $\mathrm{BaTiO}_{3}$ (Figure 1 in [15]), noting that for this system as well, remarkably good quantitative agreement with the experimentally measured values is obtained.

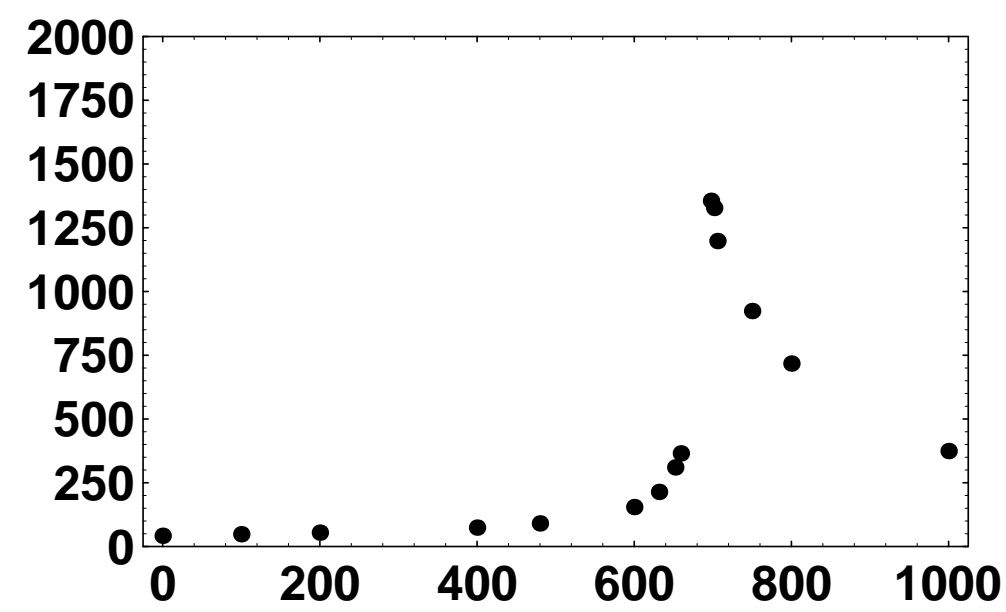

FIGURE 1. Dielectric response $\epsilon_{z z}$ as a function of temperature, in K. 


\section{LANDAU THEORY FOR THE DIVERGENT RESPONSES}

The near-divergences observed in the results described above can be already understood within a simple Landau theory which describes coupling of a scalar polar mode $\xi$ with a one-component strain $e$ and electric field $E$ :

$$
F(\xi, e ; T, E)=A \xi^{2}+B \xi^{4}-E \xi+c e^{2}+g e \xi^{2}
$$

where the temperature dependence enters through $A=A_{0}\left(T-T_{c}\right)$. If we first eliminate $e$, which is accomplished by the substitution $e \rightarrow \frac{g}{c} \xi^{2}$, the following effective fourth-order free energy results:

$$
F(\xi, e ; T, E)=A \xi^{2}+\left(B-\frac{g^{2}}{4 c}\right) \xi^{4}-E \xi
$$

Minimizing with respect to $\xi$ yields the following expressions for the dielectric susceptibility

$$
\begin{aligned}
\chi=\frac{d \xi_{\min }}{d E} & =\frac{-1}{4 A} \quad\left(T<T_{c}\right) \\
& =\frac{1}{2 A} \quad\left(T>T_{c}\right)
\end{aligned}
$$

and piezoelectric coefficient

$$
\begin{aligned}
d=\frac{d e_{\min }}{d E} & =-\frac{g}{4 A c} \sqrt{\frac{-A}{2\left(B-\frac{g^{2}}{4 c}\right)}}=-\frac{g}{c} \xi_{\min } \chi \quad\left(T<T_{c}\right) \\
& =0 \quad\left(T>T_{c}\right)
\end{aligned}
$$

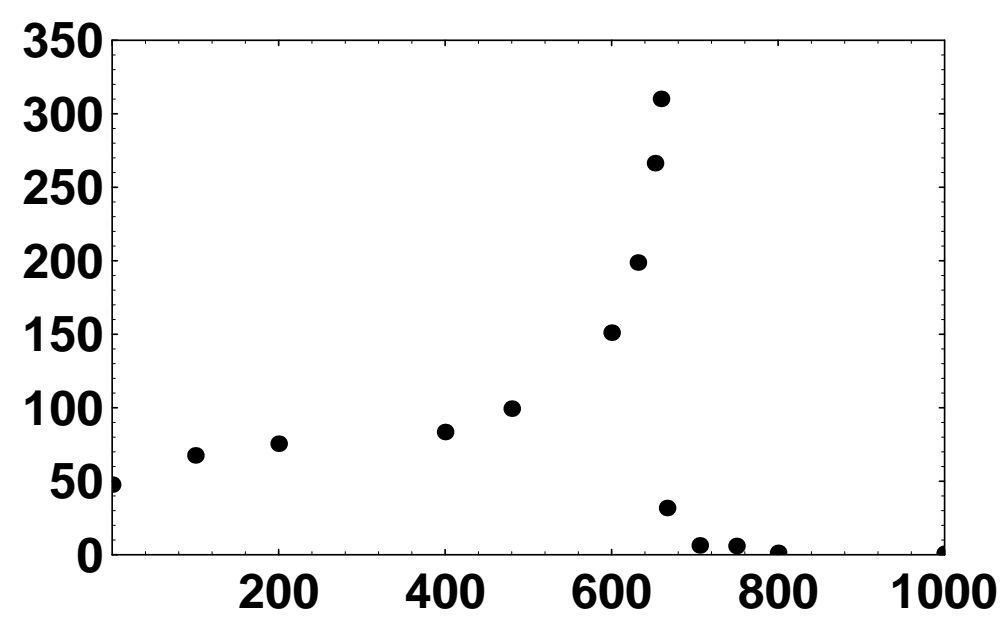

FIGURE 2. Piezoelectric response $d_{33}$ as a function of temperature, in K. 
As observed in the full simulations, $d$ diverges as $T_{c}$ is approached from below, while $\chi$ diverges on both the low and high temperature sides, and is larger above $\mathrm{T}_{c}$. In $\mathrm{PbTiO}_{3}$, these divergences are cut off due to the first-order character of the transition, so that, for example, a Curie-Weiss fit to $\chi$ above $\mathrm{T}_{c}$ yields a value of $\mathrm{T}_{0}$ about $80 \mathrm{~K}$ below $\mathrm{T}_{c}$.

\section{REAL-SPACE DECOMPOSITION OF THE CORRELATION FUNCTIONS}

The correlation function expressions for the response functions allow the interpretation of the responses in terms of the characteristic intersite correlations between the local polar distortions. Some of the correlation functions of interest have been previously calculated in effective Hamiltonian studies of $\mathrm{KNbO}_{3}$ [13] and $\mathrm{BaTiO}_{3}$ [16], allowing us to connect to and build upon the insights derived in those investigations to develop a microscopic understanding of the dielectric and piezoelectric responses.

For the dielectric response, the first term inside the parentheses of Equation 3 can be reorganized:

$$
\frac{1}{N} \sum_{i}\left(<\xi_{i \alpha} \xi_{i \beta}>+\sum_{j, n n}<\xi_{i \alpha} \xi_{j \beta}>+\sum_{j, n n n}<\xi_{i \alpha} \xi_{j \beta}>+\ldots\right)
$$

If correlations become infinite ranged, as at a second order transition, this will scale like $\mathrm{N}$, leading to a divergence in the thermodynamic limit.

For the piezoelectric tensor, we can similarly reorganize the correlation functions which appear in Equation 8, for example:

$$
\frac{1}{N} \sum_{i}\left(<\xi_{i \alpha} \xi_{i \alpha} \xi_{i \gamma}>+\sum_{j, n n}<\xi_{i \alpha} \xi_{i \alpha} \xi_{j \gamma}>+\sum_{j, n n n}<\xi_{i \alpha} \xi_{i \alpha} \xi_{j \gamma}>+\ldots\right)
$$

The real-space correlation functions appearing in the expressions above are com-

puted using fast fourier transforms of $\vec{\xi}_{i}$ and $\xi_{i \alpha} \xi_{i \beta}$. For the dielectric susceptibility, for example, the real space expressions can be written in terms of $\chi_{\alpha \beta}(\vec{q})$ as follows (taking $\vec{R}_{j}=\vec{R}_{i}+\vec{d}$ ):

$$
\begin{gathered}
\frac{1}{N} \sum_{i}<\xi_{i \alpha} \xi_{j \beta}>=\frac{1}{N} \sum_{i} \frac{1}{N^{2}} \sum_{\vec{q}, \vec{q}^{\prime}}<\xi_{\alpha}(\vec{q}) \xi_{\beta}\left(\vec{q}^{\prime}\right)>\exp \left(-i \vec{q} \cdot \vec{R}_{i}-i \vec{q}^{\prime} \cdot\left(\vec{R}_{i}+\vec{d}\right)\right) \\
=\frac{1}{N^{2}} \sum_{\vec{q}}<\xi_{\alpha}(\vec{q}) \xi_{\beta}(\vec{q})^{*}>\exp (i \vec{q} \cdot \vec{d})
\end{gathered}
$$

It can be checked that this gives the correct relation to $\chi_{\alpha \beta}$ when $\langle\vec{\xi}>=0$ :

$$
\chi_{\alpha \beta}=\frac{\beta\left(Z^{*} e a_{0}\right)^{2}}{\Omega} \sum_{\vec{d}} \frac{1}{N} \sum_{i}<\xi_{i \alpha} \xi_{j \beta}>=\sum_{\vec{d}} \frac{1}{N} \sum_{\vec{q}} \chi_{\alpha \beta}(\vec{q}) \exp (i \vec{q} \cdot \vec{d})=\chi_{\alpha \beta}(\vec{q}=0)
$$


In Figure 3, we show the real-space decomposition in the $x z$ plane of the high$\mathrm{T}$ dielectric susceptibility correlation function $\left\langle\xi_{i z} \xi_{i+\vec{d}, z}>\right.$ for $\mathrm{PbTiO}_{3}$ at two temperatures above $\mathrm{T}_{c}$. The correlation is rather isotropic, and the range of the correlations increases as expected as the temperature decreases towards $\mathrm{T}_{c}$. For comparison, the same correlation function for $\mathrm{BaTiO}_{3}$ at two temperatures above the cubic-tetragonal transition temperature is also shown in Figure 3. The highly anisotropic "chain-like" correlations discussed in previous work $[13,16]$ are clearly evident, and the correlation length transverse to the chains increases as the temperature decreases towards $\mathrm{T}_{c}$. Similar behavior is expected for $\mathrm{KNbO}_{3}$. The qualitative difference in the nature of the correlations can be directly attributed to the difference in the dispersion relation of the soft mode along $\mathrm{R}$. The implications of the different character of these correlations for the dielectric behavior of $\mathrm{PbTiO}_{3}$ and $\mathrm{BaTiO}_{3}$ are currently under investigation.

As can be seen from Equation 8, the piezoelectric response depends on thirdorder two-site correlations of the form $\left.\left.<\xi_{i \alpha}^{2} \xi_{j \beta}\right\rangle-<\xi_{i \alpha}^{2}\right\rangle\left\langle\xi_{j \beta}\right\rangle$. These have not to our knowledge been considered in previous studies. Calculations of these real-space correlations for $\mathrm{PbTiO}_{3}$ and $\mathrm{BaTiO}_{3}$ above and below the transition temperature are currently in progress.

(a)

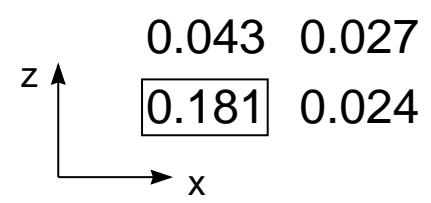

(c)
0.128

0.137

0.1640 .059

$0.217 \quad 0.064$

$0.301 \quad 0.067$ 0.4380 .065 (b) $0.008 \quad 0.008 \quad 0.008$

$0.044 \quad 0.028$

$\begin{array}{lll}0.180 & 0.026 & 0.008\end{array}$

(d)

$\begin{array}{ll}0.173 & 0.093\end{array}$

$\begin{array}{lll}0.181 & 0.094\end{array}$

$0.206 \quad 0.097$

$0.253 \quad 0.101$

$0.329 \quad 0.104$

0.4500 .102

FIGURE 3. Real-space decomposition in the $x z$ plane of the high-T dielectric susceptibility correlation function $\left\langle\xi_{i z} \xi_{i+\vec{d}, z}>\right.$ for (a) $\mathrm{PbTiO}_{3}$ at $\mathrm{T}=706 \mathrm{~K}$, (b) $\mathrm{PbTiO}_{3}$ at $\mathrm{T}=690 \mathrm{~K}$, (c) $\mathrm{BaTiO}_{3}$ at $\mathrm{T}=343 \mathrm{~K}$, and (d) $\mathrm{BaTiO}_{3}$ at $\mathrm{T}=308 \mathrm{~K}$. The numerical value in the box indicates the on-site correlation $<\xi_{i z} \xi_{i z}>$, while the numbers above and to the right of the box give the correlations for the corresponding values of $\vec{d}$. All correlations have been normalized by dividing by the square of the ground state value of $\xi_{z}\left(0.08^{2}\right.$ for $\mathrm{PbTiO}_{3}$ and $0.03^{2}$ for $\left.\mathrm{BaTiO}_{3}\right)$. Only the largest correlations are shown (above 0.0078 for $\mathrm{PbTiO}_{3}$ and above 0.056 for $\mathrm{BaTiO}_{3}$ ). 


\section{DISCUSSION AND CONCLUSIONS}

As already discussed, the use of an effective Hamiltonian for calculating the dielectric and piezoelectric response involves a number of approximations. The effects of thermal expansion and the neglect of other polar modes cannot be incorporated without adding additional degrees of freedom to the model (unless one is willing to accept semi-empirical input). However, the low-order expansion around the cubic reference structure can be improved, which in particular will yield better model results for the properties of the $\mathrm{PbTiO}_{3}$ tetragonal ground state [19]. This could involve both $\mathcal{H}_{\text {eff }}$ and $P$. In the case of the polarization $P$, the next order terms involve including strain dependence and $\xi$ dependence of the effective charges. First-principles calculations $[17,18]$ suggest that the former corrections are rather small compared to the latter. Calculations for these refinements for $\mathrm{PbTiO}_{3}$ are currently in progress.

Since the effective Hamiltonian approach is limited to the description of soft-mode contributions to the responses, we focus on the near-divergent responses where these contributions strongly dominate. Fortunately, this regime is, in any case, the one of greatest interest for understanding the origin of and engineering large responses in a variety of systems characterized by proximity to a lattice instability. Further insight can be gained from the real-space decomposition of the divergent responses based on their expressions in terms of correlation functions.

Finally, these results for pure systems suggest an interpretation for the origin of large responses in solid solutions. For such systems, there is a distribution of local environments, and for a given temperature some subset of lattice degrees of freedom will be marginally stable. This idea has been investigated using first principles results in $\mathrm{Pb}_{1-x} \mathrm{Ge}_{x} \mathrm{Te}$, and more details are presented in Refs. [20,21].

\section{ACKNOWLEDGMENTS}

We thank R. E. Cohen, A. Garcia, R. M. Martin, D. Vanderbilt, and U. V. Waghmare for helpful discussions. This work was supported by ONR grant N0001497-1-0047 and the Alfred P. Sloan Foundation, and was in part carried out at the Aspen Center for Physics.

\section{REFERENCES}

1. J. M. Herbert, Ferroelectric Transducers and Sensors, New York: Gordon and Breach, 1982.

2. J. P. Remeika and A. M. Glass, Mater. Res. Bull. 5, 37 (1970).

3. K. Kakuta, T. Tsurumi and O. Fukunaga, Jpn. J. Appl. Phys. 34, 5341 (1995).

4. Z. Li, M. Grimsditch, C. M. Foster and S.-K. Chan, J. Phys. Chem. Solids 57, 1433 (1996).

5. C. J. Johnson, Appl. Phys. Lett. 7, 221 (1965). 
6. F. Jona and G. Shirane, Ferroelectric Crystals, New York: Macmillan, 1962.

7. K. M. Rabe and U. V. Waghmare, Phys. Rev. B52, 13236 (1995).

8. K. M. Rabe and U. V. Waghmare, J. Phys. Chem. Solids 57, 1397 (1997).

9. U. V. Waghmare and K. M. Rabe, Phys. Rev. B55, 6161 (1997).

10. W. Zhong, D. Vanderbilt, and K. M. Rabe, Phys. Rev. Lett. 73, 1861 (1994); Phys. Rev. B 52, 6301 (1995).

11. J. F. Nye, Physical Properties of Crystals, Oxford: Clarendon, 1964.

12. A. Garcia and D. Vanderbilt, these proceedings.

13. H. Krakauer, R. Yu, C.-Z. Wang, and C. LaSota, Ferroelectrics, in press; H. Krakauer, R. Yu, C.-Z. Wang, K. M. Rabe and U. V. Waghmare, cond-mat/9710088.

14. Z. Li, M. Grimsditch, X. Xu and S.-K. Chan, Ferroelectrics 141, 313 (1993).

15. A. Garcia and D. Vanderbilt, cond-mat/9801177.

16. J. Padilla, W. Zhong and D. Vanderbilt, mtrl-th/9509005.

17. C.-Z. Wang, R. Yu, and H. Krakauer, Phys. Rev. B54, 11161 (1996).

18. Ph. Ghosez, X. Gonze, Ph. Lambin and J.-P. Michenaud, Phys. Rev. B51, R6765 (1995).

19. U. V. Waghmare, unpublished.

20. E. Cockayne and K. M. Rabe, cond-mat/9712232.

21. E. Cockayne and K. M. Rabe, these proceedings. 